\title{
Nim is easy, chess is hard — but why??
}

\author{
Aviezri S. Fraenkel* \\ June 27, 2006 \\ Department of Computer Science and Applied Mathematics \\ Weizmann Institute of Science \\ Rehovot 76100, Israel
}

In nim, a finite number of piles of finitely many tokens is given. Two players alternate in selecting a pile and removing from it any positive number of tokens, possibly the entire pile. The player first unable to move loses and the opponent wins. Nim is easy. Why? Because it has an easy strategy: write the number of tokens in each pile in binary, and "add" them without carry, an addition also known as XOR (Exclusive Or). If the sum is 0, you better be a gentle(wo)man and offer your opponent to move first, because you can win as second player. If the sum is nonzero you can move first and make it 0 , thus winning.

Chess is difficult. Why? Lewis Dartnell wrote in "Practice makes perfect", Plus, Issue 28 January 2004, comparing chess with nim: "Chess, however, is almost inconceivably more complex, and the pieces can be arranged on the 64 squares of the board in 1044 distinct ways. One mathematician has calculated that there are about $10^{\left(10^{50}\right)}$ different legal games, which is far more than the number of particles in the entire visible universe. This is effectively an infinite number of permutations, and so in all practical senses it is impossible to play chess perfectly."

A similar reason is given by Marianne Freiberger, in her review of "Luck, logic and white lies", Plus, Issue 35 May 2005: "In combinatorial games such as chess, the number of possible combinations of moves is astronomical, meaning that a complete analysis is totally unfeasible."

In J. Recreational Math., 19:2, pp. 119-125, 1987, Steven Goldberg wrote, "There is a way to play chess so that you never lose: the good news is that this has been proved. The bad news is that all the computers in the world will never be able to discover it". The latter claim is based on the statement that "the number of possible moves in chess has been estimated to be approximately $12 \times 10^{81}$ ".

Incidentally, the difference in the estimates of Dartnell and Goldberg is in itself super-astronomical. But the former refers to the number of games, whereas we are interested in the number of moves. Therefore we stick with Goldberg's estimate.

\footnotetext{
*fraenkel@wisdom.weizmann.ac.il http://www.wisdom.weizmann.ac.il/ fraenkel
} 
The astronomical number of moves of chess was also given as the reason for its computational intractability in a recent book on the fundamentals of computer science.

We thus have at least four authors who stated that chess is computationally intractable because of the large number of its moves. The reasoning has been voted correct unanimously.

The logic is flawed, however. It is not hard to see that the number of positions of a game of nim with $m$ piles of size at most $n$, is the number of combinations of $n$ out of $m$. For the case $m=n$ this yields about $4^{n} / \sqrt{n}$ positions. Thus for $n=140$, i.e., 140 piles, each of size at most 140 , the number of positions of nim is more than 10 times the above number of estimated moves of chess. Yet it's trivial to compute the strategy for any such nim game, and a computer can do it in at most a few seconds.

The point is that an efficient strategy goes about it intelligently: it homes in onto the optimal moves without going through all possible moves! This observation raises the question whether perhaps also chess has an efficient strategy, and we simply haven't yet found it. In the rest of this note we will show that this is highly unlikely.

1. In nim no repeated positions are possible, whereas chess admits cycles. In nim there are no capture moves, which abound in chess. Nim is impartial, i.e., the set of moves from any position is the same for both players, whereas chess is partizan: the black player cannot move any white piece, and conversely. Nim has only one terminating state, when all piles are empty. But in chess, every checkmating configuration is a terminating state, and they abound. Moreover, for solving any large system, we strive to decompose it into a number of smaller tasks, and solve each one individually. This can be done for nim, since the piles are distinct. But chess does not seem to decompose into disjoint parts (except some very simple configurations consisting of pawns and the two kings only). Thus chess appears to be considerably more complex than nim.

2. Chess has been proved to be "Exptime-complete" [1]. This statement roughly says the following: define chess on a general $n \times n$ board in any reasonable way, with one white and one black king. Then every conceivable or inconceivable algorithm to decide who can win from an arbitrary mid-chessposition, must necessarily take exponential time in the size of the position!

An exponential function has the form $c^{n}$ where $c>1$ is a constant, such as 2. A polynomial function has the form $n^{c}$, with $c>1$ a constant. Exponential functions grow very fast ("exponentially"), whereas polynomial functions grow at a moderate rate. Thus $2^{20}$ is already above one million, whereas $20^{2}=400$. The reader is encouraged to construct a table of the values $2^{n}$ and $n^{2}$ for all $n$ at least up to 40 . ( $2^{40}$ is bigger than a thousand billion $\left(10^{12}\right)$, whereas $40^{2}=1,600$.)

It may be helpful to view the difference between exponential and polynomial functions from a cosmological point of view. Estimates by astrophysicists of the number of particles in the observable universe are currently (2005) on the order of $10^{85}<2^{299}$ [2], but $299^{2}$ is only 89,401 .

These type of considerations motivate the following convention used in com- 
putational complexity. A problem that admits a polynomial time algorithm is called tractable; otherwise it is intractable.

In conclusion, the provable intractability of chess doesn't exclude the possibility that $8 \times 8$ chess has an easy perfect solution, which somehow depends on the number 8 or other small numbers, though this seems to be unlikely. Moreover, it is likely that the world-wide efforts to improve the traditional $8 \times 8$ computer-chess programs will lead to more Gary Kasparov and other worldchess-champions defeats.

Yet none of these Turing-computer programs can ever determine with certainty who can win from an arbitrary mid-chess-position in $n \times n$ chess for general $n$. The reason for this is the inherent complexity of $n \times n$ chess as reflected by its Exptime-completeness - not the astronomical number of its moves. It is true that the high complexity of $n \times n$ chess implies that it has a very large number of moves, but not conversely! And also the large number of moves of $8 \times 8$ chess, doesn't imply that it is highly complex, which is the main message of this note.

\section{References}

[1] A. S. Fraenkel and D. Lichtenstein [1981], Computing a perfect strategy for $n \times n$ chess requires time exponential in $n, J$. Combin. Theory (Ser. A) 31, 199-214.

[2] http://en.wikipedia.org/wiki/Wikipedia:Size_comparisons 